DTP $/ 95 / 88$

October 1995

\title{
Fermiophobic Higgs bosons at the Tevatron
}

\author{
A.G. Akeroyd \\ Department of Mathematical Sciences, Centre for Particle Theory, \\ University of Durham, \\ Durham DH1 3LE, England.
}

\begin{abstract}
Higgs bosons with negligible couplings to fermions can arise in various nonminimal Higgs sectors. We show that such a particle could be discovered during the current run at the Tevatron, and would be evidence against a minimal supersymmetric Higgs sector.
\end{abstract}

\footnotetext{
${ }^{1}$ A.G.Akeroyd@durham.ac.uk
} 


\section{Introduction}

The Standard Model (SM) [1] has proved remarkably successful to date in describing the particle interactions of nature. However, the theory requires that the electroweak symmetry is broken and an efficient way of accomplishing this is to introduce scalar particles (Higgs bosons) with non-zero vacuum expectation values (VEVs) [2]. Thus far no such particles have been detected and therefore it is prudent to explore all possible Higgs sectors. The minimal SM consists of one complex isospin Higgs doublet which after symmetry breaking predicts one physical neutral scalar $\left(\phi^{0}\right)$, although much can be found in the literature concerning extended models [3] i.e. the nonminimal SMl . Extended Higgs sectors with additional doublets/triplets always require exotic Higgs bosons with electric charge $\left(H^{ \pm}\right)$and zero tree-level couplings to gauge bosons $\left(A^{0}\right)$. Also possible in some extended models is 'fermiophobia' [4], [5] i.e. zero tree-level couplings to fermions. Such particles $\left(H_{F}\right)$ can only arise in certain Higgs models, and in particular are not predicted by the minimal supersymmetric model (MSSM). Therefore the discovery of a $H_{F}$ would be evidence against a minimal supersymmetric extended Higgs sector.

Our work is organised as follows. In Section 2 we describe the various Higgs models which can contain fermiophobia, and then investigate the properties of $H_{F}$. Section 3 deals with the phenomenology of $H_{F}$ at both the Tevatron and proposed Tevatron upgrade. Finally Section 4 contains our conclusions.

\section{Models with Fermiophobia}

The most theoretically favourable non-minimal Higgs sectors are those that contain only doublet representations. These naturally keep $\rho \equiv M_{W}^{2} /\left(M_{Z}^{2} \cos ^{2} \theta_{W}\right) \approx 1$ [6]. Models with triplets can also be considered and the most popular of these was proposed by Georgi and Machacek containing one-doublet and two-triplets [7], [8], [9]. In this paper we shall consider the various two-Higgs-doublet models (2HDM) of which there are four distinct versions [10], and the above mentioned Higgs triplet model (HTM).

\begin{tabular}{|c|c|c|c|c|}
\hline & Model I & Model I' & Model II & Model II' \\
\hline $\mathrm{u}$ (up-type quarks) & 2 & 2 & 2 & 2 \\
\hline $\mathrm{d}$ (down-type quarks) & 2 & 2 & 1 & 1 \\
\hline $\mathrm{e}$ (charged leptons) & 2 & 1 & 1 & 2 \\
\hline
\end{tabular}

Table 1: The four distinct structures of the 2HDM.

Table 1 shows the four different ways with which the 2HDM can be coupled to the fermions. The numbers (1 or 2 ) show which Higgs doublet couples to which fermion

\footnotetext{
${ }^{2}$ Defined by assuming no other new particles apart from Higgs bosons.
} 
type. Natural flavour conservation [11] requires that at most one doublet can couple to any particular fermion-type. Model II is the structure required for the MSSM [3], [12] and thus it has received substantially more attention in the literature. Model I is the only model that can display fermiophobia and this becomes clear when we view the couplings in Table 2. We are interested here in the lighter of the two neutral, $\mathrm{CP}$-even Higgs bosons $(h)$.

\begin{tabular}{|c|c|c|c|c|}
\hline & Model I & Model I' & Model II & Model II $^{\prime}$ \\
\hline$h u \bar{u}$ & $\cos \alpha / \sin \beta$ & $\cos \alpha / \sin \beta$ & $\cos \alpha / \sin \beta$ & $\cos \alpha / \sin \beta$ \\
\hline$h \bar{d}$ & $\cos \alpha / \sin \beta$ & $\cos \alpha / \sin \beta$ & $-\sin \alpha / \cos \beta$ & $-\sin \alpha / \cos \beta$ \\
\hline$h e \bar{e}$ & $\cos \alpha / \sin \beta$ & $-\sin \alpha / \cos \beta$ & $-\sin \alpha / \cos \beta$ & $\cos \alpha / \sin \beta$ \\
\hline
\end{tabular}

Table 2: The fermion couplings of $h$ in the 2HDM relative to those for the minimal SM Higgs boson $\left(\phi^{0}\right)$.

Here $\alpha$ is a mixing angle used to diagonalize the $\mathrm{CP}$-even mass matrix and $\beta$ is defined by $\tan \beta=v_{2} / v_{1}$ ( $v_{i}$ is the VEV of the $i^{\text {th }}$ doublet and $\left.v_{1}^{2}+v_{2}^{2}=246 \mathrm{GeV}^{2}\right)$. From Table 2 we see that fermiophobia is only possible in Model I if $\cos \alpha \rightarrow 0$ [4]. We note that the heavier CP-even Higgs $(H)$ in Model I would itself be fermiophobic if $\cos \alpha \rightarrow 1$. However this particle could be substantially heavier than $h$ and so is not considered. From now on we shall label the fermiophobic Higgs in this model as being $h$, with $H_{F}$ referring to any generic fermiophobic Higgs. Therefore it is apparent that fermiophobia is not possible in the MSSM since it requires Model II type couplings. Hence searching for $H_{F}$ is well motivated. We note that another signal of the 2HDM (Model I) which is not possible in the MSSM would be the discovery of a light $H^{ \pm}$ $\left(M_{H^{ \pm}} \leq M_{W}\right)$; this is possible through direct pair production at LEP2 [13] or top quark decay at the Tevatron [14].

The other model that we shall study and contains fermiophobia is the HTM. Predicted here are two fermiophobic neutral bosons, $H_{5}^{0}$ and $H_{1}^{0^{\prime}}$. In this paper we shall not consider a charged $H_{F}$; for recent studies of the latter we refer the reader to Refs. [15], [16]. In an earlier paper [17] we analysed this model using a natural argument of equating all Higgs self couplings $\left(\lambda_{i}\right)$ to 1 ; it was shown that $H_{1}^{0^{\prime}}$ can be taken as a physical mass eigenstate and we also obtained the following natural mass hierarchy (with $v^{2}=246 \mathrm{GeV}^{2}$ ):

$$
M_{\psi_{1}}^{2}=10 v^{2} \rightarrow 16 v^{2}, \quad M_{H_{5}}^{2}=3 v^{2}, \quad M_{H_{3}}^{2}=v^{2}, \quad M_{\psi_{2}}^{2}=0 \rightarrow 1.5 v^{2} .
$$

The compositions of the mass eigenstates $\psi_{1}$ and $\psi_{2}$ are given by

$$
\begin{aligned}
& \psi_{1}=H_{1}^{0^{\prime}} \sin \alpha_{T}+H_{1}^{0} \cos \alpha_{T}, \\
& \psi_{2}=H_{1}^{0^{\prime}} \cos \alpha_{T}-H_{1}^{0} \sin \alpha_{T},
\end{aligned}
$$

with $H_{1}^{0}$ being a neutral scalar similar to that of the minimal SM, and $\alpha_{T}$ being a mixing angle. Ref. [17] shows that $\sin \alpha_{T} \leq 0.05$ or $0.999 \leq \sin \alpha_{T} \leq 1$, and so 
negligible mixing occurs in Eqs. (2) and (3). Therefore $H_{1}^{0^{\prime}}$ could be the lightest or the heaviest of the bosons in the HTM depending on the exact value of the angle $\alpha_{T}$. We shall be concentrating on the scenario of it being the lightest but will also mention detection prospects if this is not the case. Ref. [17] constrains $\alpha_{T}$ by using the bound $\sin \theta_{H} \leq 0.63,3$ found from considering the effects of $H_{3}^{ \pm}$on the $Z \rightarrow b \bar{b}$ vertex. This result is for $M_{H_{3}} \leq 200 \mathrm{GeV}$ which we see as being justified if one wishes to search for $H_{1}^{0^{\prime}}$ at the Tevatron; from the ratios in Eq. (1) we see that $M_{H_{3}} \leq 200$ GeV would imply $0 \leq M_{\psi_{2}} \leq 245 \mathrm{GeV}$, which is the mass range that is relevant at the Tevatron. The other neutral fermiophobic Higgs, $H_{5}^{0}$, is likely to be heavier than $H_{1}^{0^{\prime}}$ (if $\psi_{2} \equiv H_{1}^{0^{\prime}}$ ) and we shall see that it is harder to produce at the Tevatron due its more suppressed couplings to vector bosons. In Ref. [17] we proposed that the detection of a $H_{F}$ would suggest the HTM, since the 2HDM (Model I) requires fine-tuning for fermiophobia.

It is possible to apply the above natural argument to the 2HDM (Model I) to see the variation of $\sin \alpha$ with $\tan \beta$. Plotted in Figure 11 is $\sin 2 \alpha$ as a function of $v_{2}$. We see that maximal mixing $\left(\sin 2 \alpha=1, \alpha=45^{\circ}\right)$ occurs when $v_{2}=v_{1} \approx 174 \mathrm{GeV}$. For $v_{2} \gg 174 \mathrm{GeV}$ (i.e. $\tan \beta \gg 1$ ), the two $\alpha$ solutions for $\sin 2 \alpha$ approach $0^{\circ}$ and $90^{\circ}$. Hence for fermiophobia $\left(\alpha \rightarrow 90^{\circ}\right)$ this argument would require larger $\tan \beta$, a result consistent with the bound $\tan \beta \geq 1.25$ for $M_{H^{ \pm}} \leq 200 \mathrm{GeV}$ [18].

We may also obtain the analogous mass hierarchy (see Eq. (1)) for the 2HDM (Model I). Figure 2 shows the squared masses of $H$ and $h$ as a function of $v_{2}$, and from this we see that $7 v^{2} \leq M_{H}^{2} \leq 8 v^{2}$ and $v^{2} \leq M_{h}^{2} \leq 2 v^{2}$. Therefore we have

$$
M_{H}^{2}=7 v^{2} \rightarrow 8 v^{2}, \quad M_{h}^{2}=v^{2} \rightarrow 2 v^{2}, M_{H^{ \pm}}^{2}=v^{2}, \quad M_{A^{0}}^{2}=v^{2} .
$$

Eq. (4) suggests that $h$ is likely to be of comparable mass to $M_{H^{ \pm}}$and so justifies the use of the bound $\tan \beta \geq 1.25$ for $M_{H^{ \pm}} \leq 200 \mathrm{GeV}$, if one wishes to search for $h$ at the Tevatron.

We now study the branching ratios $(\mathrm{BRs})$ of $H_{F}$. Tree-level decays to fermions are obviously not allowed, and if $M_{H_{F}} \leq 80 \mathrm{GeV}$ then the only possible tree-level channels are $H_{F} \rightarrow W^{*} W^{*}, Z^{*} Z^{*}$, with '*' denoting an off-shell vector bosont. Since these latter decays are not very strong (the vector bosons being considerably off-shell) then one-loop mediated decays can compete and these are displayed in Figure 3. For the case of $H_{F} \rightarrow \gamma \gamma$, the $W$ mediated decays give the dominant contribution [3], 19] and only these are included . The one-loop decays to $f \bar{f}$ are renormalization scheme dependent and it is conventional in the literature to consider an extreme fermiophobic Higgs with the renormalized $H_{F} \rightarrow f \bar{f}$ vertex set equal to zero [20], [21]. The BRs predicted by Refs. [20] and 21] agree and imply that the channel $H_{F} \rightarrow \gamma \gamma$ dominates

\footnotetext{
${ }^{3} \sin \theta_{H}$ is the analogy of $\tan \beta$ for the HTM, defined by $\sin \theta_{H}=\sqrt{8 b^{2}} / \sqrt{a^{2}+8 b^{2}}$, with the doublet (triplet) VEV denoted by a (b).

${ }^{4}$ Not including decays to other Higgs bosons which will be heavily off-shell also.
} 


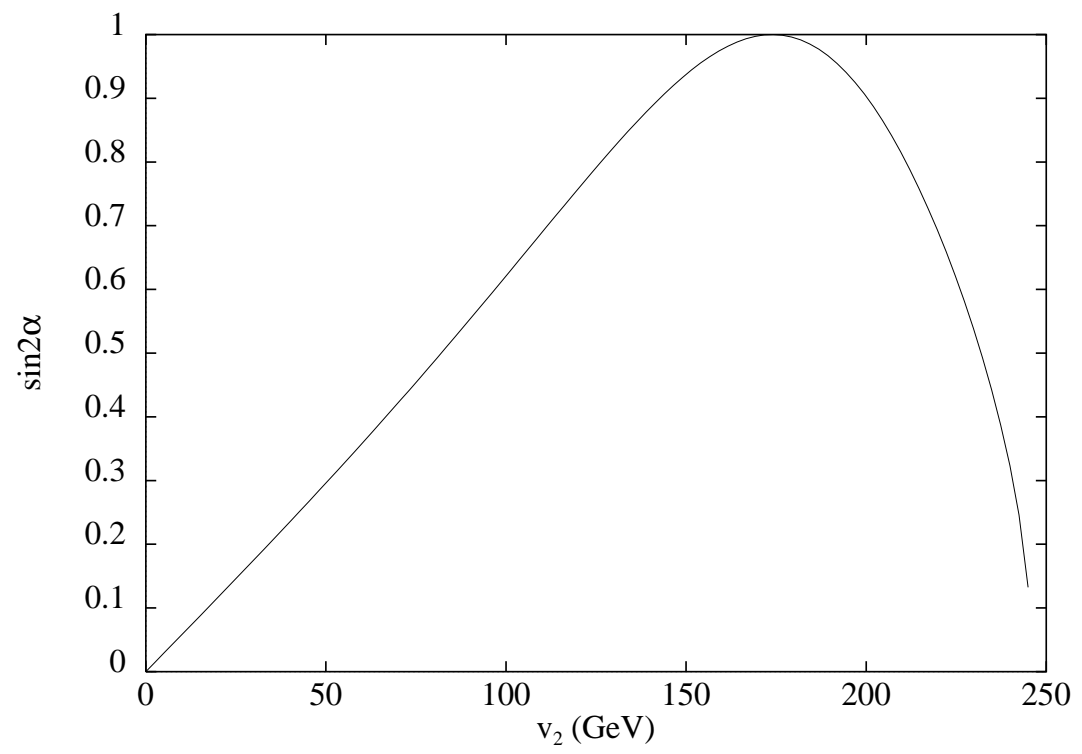

Figure 1: $\sin 2 \alpha$ as a function of $v_{2}$.

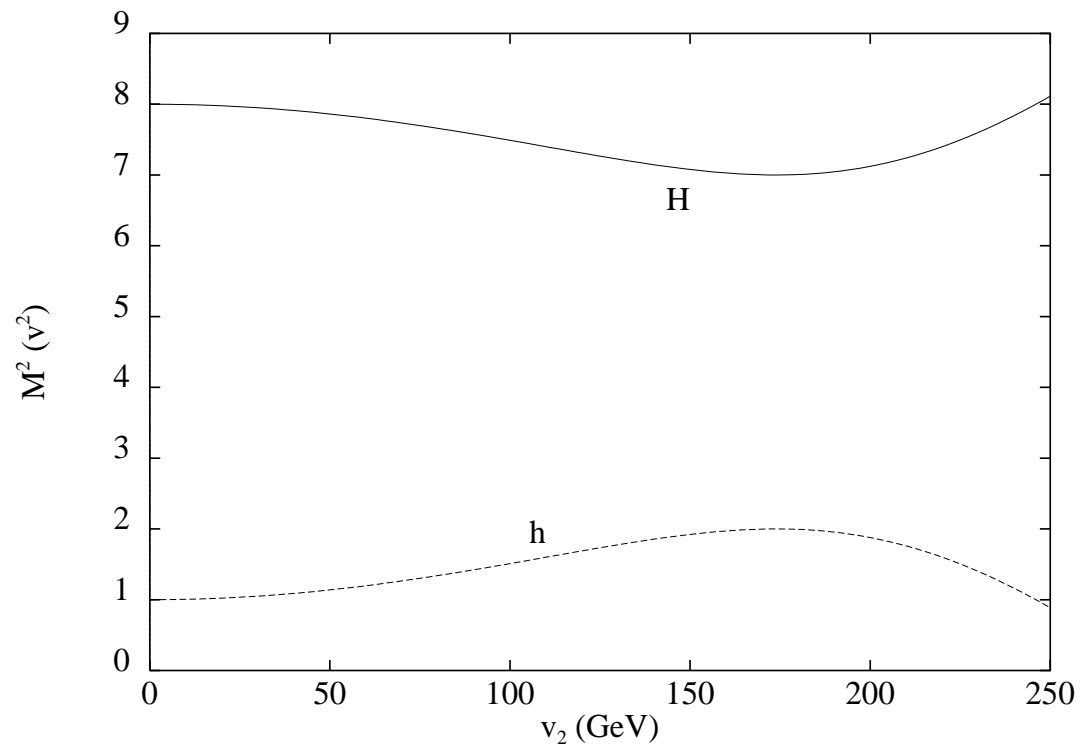

Figure 2: The squared masses of $H$ and $h$ as a function of $v_{2}$ 
for $M_{H_{F}} \leq 80 \mathrm{GeV}$; at $M_{H_{F}} \approx 95 \mathrm{GeV}$ the tree-level process $H_{F} \rightarrow W W^{*}$ is equally likely as $H_{F} \rightarrow \gamma \gamma$, each having $\mathrm{BR}=45 \%$. In contrast, for $\phi^{0}$ and the lightest neutral $\mathrm{CP}-$ even scalar of the MSSM the branching ratio to two photons is of the order $0.1 \%$. For higher $M_{H_{F}}$ the vector boson channels dominate along with decays to other Higgs bosons $\left(H_{F} \rightarrow t \bar{t}\right.$ is not allowed at tree-level). Therefore the distinctive fermiophobic signature of $H_{F} \rightarrow \gamma \gamma$ is disappearing for $M_{H_{F}} \geq 100 \mathrm{GeV}$, and so we shall focus on the region of $M_{H_{F}} \leq 100 \mathrm{GeV}$. For the heavier mass region $(\geq 160 \mathrm{GeV}$ ) the only difference between the decays of $H_{F}$ and $\phi^{0}$ would be due to the presence of lighter Higgs bosons e.g. $h \rightarrow A^{0} Z$.
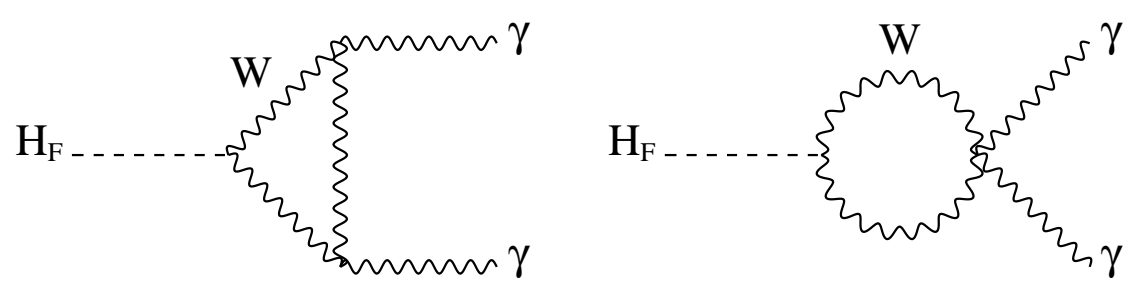

Figure 3: Two-photon decay of $H_{F}$.

The BRs used in Ref. [20] are for a $H_{F}$ with $\phi^{0}$ strength (i.e. minimal SM strength) couplings to vector bosons. This is not the case for the $H_{F}$ that we are considering, as can be seen from Eqs. $(5 \rightarrow 7)$. The couplings here are expressed relative to those of the minimal SM Higgs boson (with $s_{H} \equiv \sin \theta_{H}$ ) $[8]$ :

$$
\begin{array}{cc}
H_{1}^{0^{\prime}} W^{+} W^{-}: \frac{2 \sqrt{2}}{\sqrt{3}} s_{H}, & H_{1}^{0^{\prime}} Z Z: \frac{2 \sqrt{2}}{\sqrt{3}} s_{H}, \\
H_{5}^{0} W^{+} W^{-}: \frac{1}{\sqrt{3}} s_{H}, & H_{5}^{0} Z Z: \frac{-2}{\sqrt{3}} s_{H}, \\
h W^{+} W^{-}:-\cos \beta, & h Z Z:-\cos \beta .
\end{array}
$$

Eqs. (5) and (7) show that both the $H_{F} W^{+} W^{-}$and $H_{F} Z Z$ couplings for $H_{1}^{0^{\prime}}$ and $h$ are scaled by the same amount, and so the BRs used in Ref. 20 can be used. This is not true for $H_{5}^{0}$ which has an enhanced $H_{5}^{0} Z Z$ compared to $H_{5}^{0} W^{+} W^{-}$. However, in the region of $M_{F} \leq 80 \mathrm{GeV}$ (which is of interest to us) the channel $H_{5}^{0} \rightarrow Z^{*} Z^{*}$ is small and so we may use the results in Ref. [20] to a very good approximation.

\section{Phenomenology at the Tevatron}

For $\phi^{0}$ the main production process at the Tevatron $(\sqrt{s}=1.8 \mathrm{TeV})$ is gluon-gluon fusion via a top quark loop [22]. This is not allowed for $H_{F}$ and nor are any diagrams 
involving associated production with top quarks [23], [24]. Therefore there remains two processes; associated production with vector bosons [25] and vector boson fusion [26]. However, Ref. [20] shows that the latter gives less events and so we shall focus on the former whose Feynman diagram is displayed in Figure 4 . As mentioned in Section 2, Ref. 20] assumed minimal SM strength couplings to vector bosons for $H_{F}$ and so the production cross sections for $H_{1}^{0^{\prime}}, H_{5}^{0}$ and $h$ relative to those for $\phi^{0}$ will scale by the squares of the couplings given in Eqs. $(5 \rightarrow 7)$. Thus for the process $q \bar{q} \rightarrow W^{*} \rightarrow W H_{F}$ we have the following cross section ratios:

$$
H_{5}^{0}: H_{1}^{0^{\prime}}: h: \phi^{0}=\frac{1}{3} s_{H}^{2}: \frac{8}{3} s_{H}^{2}: \cos ^{2} \beta: 1,
$$

and for $q \bar{q} \rightarrow Z^{*} \rightarrow Z H_{F}$

$$
H_{5}^{0}: H_{1}^{0^{\prime}}: h: \phi^{0}=\frac{4}{3} s_{H}^{2}: \frac{8}{3} s_{H}^{2}: \cos ^{2} \beta: 1 .
$$

Due to the bounds $\sin ^{2} \theta_{H} \leq 0.39$ and $\cos ^{2} \beta \leq 0.39$ we see that $H_{1}^{0^{\prime}}$ may be produced with $\phi^{0}$ strength in both channels, while $h$ has at best a cross section 0.39 that of $\phi^{0}$. $H_{5}^{0}$ has very weak couplings to $W^{+} W^{-}$(at best 0.13 that of $\phi^{0} W^{+} W^{-}$), but better to $Z Z$.

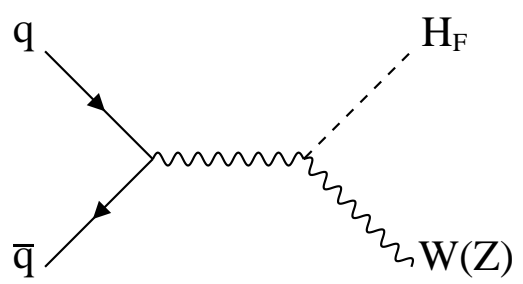

Figure 4: The main production mechanism of $H_{F}$ at the Tevatron.

We note that a $H_{F}$ with $\phi^{0}$ strength couplings to $Z Z$ would have been seen at LEP if $M_{H_{F}} \leq 60 \mathrm{GeV}$ [27]. Eq. (9) shows that this lower bound will in general be weaker for $H_{1}^{0^{\prime}}, h$ and $H_{5}^{0}$. The method of searching for $H_{F}$ at the Tevatron is described in Ref. [20 and we shall briefly review it here. The photons from $H_{F}$ act as a trigger for the events, and then various cuts are applied depending on whether the vector bosons decay hadronically or leptonically. For the leptonic decay it is shown that the main background ( $W \gamma \gamma$ and $Z \gamma \gamma$ ) is negligible. Hence we only require a reasonable number of events $(\geq 3)$ in this channel for detection. For the hadronic decays of the vector bosons there is a background $(j j \gamma \gamma)$. are combined due to the invariant mass distribution being unable to separate the $W$

\footnotetext{
${ }^{5}$ From processes like $g g, q q \rightarrow q q \gamma \gamma$.
} 
and $Z$ peaks. Table 3 shows the expected number of signal and background events 9 for $67 \mathrm{pb}^{-1}$ of data, which is the current data sample at the Tevatron. The numbers are for $H_{1}^{0^{\prime}}$ with $s_{H}^{2}=0.39$, its maximum value.

\begin{tabular}{|c|c|c|c|}
\hline$M_{H_{F}}(\mathrm{GeV})$ & $W H / Z H$ (leptonic) & $W H / Z H$ (jets) & $j j \gamma \gamma$ \\
\hline 60 & $9.8 / 7.7$ & 50.9 & 3.5 \\
\hline 80 & $3.7 / 3.5$ & 20.2 & 1.9 \\
\hline 100 & $0.6 / 0.5$ & 3.1 & 1.0 \\
\hline
\end{tabular}

Table 3: Number of signal and background events for the process $q \bar{q} \rightarrow W^{*}\left(Z^{*}\right) \rightarrow H_{1}^{0^{\prime}} W(Z)$, with $H_{1}^{0^{\prime}} \rightarrow \gamma \gamma$ and $W \rightarrow l \nu, Z \rightarrow l \bar{l}, \nu \bar{\nu}$, or $W, Z \rightarrow j j$.

From Table 3 we see that the region $M_{H_{F}} \leq 80 \mathrm{GeV}$ can be covered with $\geq 3$ events in the background free leptonic channel, and $\mathrm{a} \geq 4.3 \sigma$ signal in the hadronic channel. With $140 \mathrm{pb}^{-1}$ available by the end of 1995 the event numbers in Table 3 will be increased by a factor of approximately 2.1. This would enable the region $M_{H_{F}} \leq 90$ $\mathrm{GeV}$ to be covered, i.e. the mass at which the $\gamma \gamma$ decay starts to fall rapidly. It is very possible that $s_{H}^{2}$ is considerably less than 0.39 , and if this is the case then the signal becomes weaker. With $140 \mathrm{pb}^{-1}$ of luminosity and $M_{H_{F}}=60$ (80) GeV one can obtain $\geq 3$ events in the leptonic channel if $s_{H}^{2} \geq 0.06\left(s_{H}^{2} \geq 0.16\right)$.

For the case of $h$ the maximum number of signal events is less due to the cross section being proportional to $\cos ^{2} \beta$. Table 4 is the analogy of Table 3 for $h$ with $\cos ^{2} \beta=0.39$.

\begin{tabular}{|c|c|c|c|}
\hline$M_{H_{F}}(\mathrm{GeV})$ & $W H / Z H$ (leptonic) & $W H / Z H($ jets $)$ & $j j \gamma \gamma$ \\
\hline 60 & $3.7 / 2.9$ & 19.1 & 3.5 \\
\hline 80 & $1.4 / 1.3$ & 7.6 & 1.9 \\
\hline 100 & $0.2 / 0.2$ & 1.2 & 1.0 \\
\hline
\end{tabular}

Table 4: Same as for Table 3 but for the process $q \bar{q} \rightarrow W^{*}\left(Z^{*}\right) \rightarrow h W(Z)$.

We see that $M_{F} \leq 60 \mathrm{GeV}$ can be probed ( $\geq 3$ events in the leptonic channel and $\mathrm{a} \geq 4 \sigma$ signal in the hadronic channel). The coverage increases to $M_{F} \leq 80 \mathrm{GeV}$ with $140 \mathrm{pb}^{-1}$.

Would it be possible to distinguish between $H_{0}^{1^{\prime}}$ and $h$ ? If $s_{H}^{2}$ is near its maximum of 0.39 then the cross section for a given $M_{F}$ is considerably larger for $H_{0}^{1^{\prime}}$ than that for $h$ (see Eqs. (8) and (9)). Once the mass of $H_{F}$ is measured one can estimate the cross section and thus distinguish between the two models. Of course a sufficient

\footnotetext{
${ }^{6}$ The event numbers in all our tables are obtained from Ref. [20] with appropriate scaling for a particular Higgs model.
} 
number of $\gamma \gamma$ events will be needed to measure the mass and so one should use the hadronic channel. Sufficient events should be present, certainly up to $M_{F} \approx 80 \mathrm{GeV}$.

The above analysis has assumed that the lighter mass eigenstate $\psi_{2}$ is composed dominantly of $H_{0}^{1^{\prime}}$ (see Eq. (3)). If this is not the case then $\psi_{2} \approx H_{1}^{0}$ and the heavier eigenstate $\psi_{1}$ will be equal to $H_{0}^{1^{\prime}}$. Therefore $H_{5}^{0}$ will be the lighter $H_{F}$ in the HTM. If $M_{H_{5}^{0}} \leq 90 \mathrm{GeV}$ then one may search for the $\gamma \gamma$ decays at the Tevatron, and the mass hierarchy (Eq. (1)) would suggest that $H_{3}^{0}, H_{3}^{ \pm}$, and $H_{1}^{0}$ would also be light. However, Ref. [20] shows that at least $1000 \mathrm{pb}^{-1}$ of luminosity would be needed to search for the SM Higgs $\left(\phi^{0}\right)$, and so more would be needed for $H_{1}^{0}$ which has $\phi^{0}$ strength couplings only in the limit of $s_{H} \rightarrow 0$. The three-plet bosons $\left(H_{3}^{0}\right.$ and $\left.H_{3}^{ \pm}\right)$ would be difficult to detect at the Tevatron and prospects are much better at LEP2. The doubly charged Higgs $\left(H_{5}^{ \pm \pm}\right)$is likely to have a similar mass to $H_{5}^{0}$ (the five-plet members are degenerate at tree-level) and would offer the best signature of the HTM. Returning to $H_{5}^{0}$, we find that if $s_{H}^{2}=0.39$ then $\geq 3$ events are predicted in the $Z^{*} \rightarrow Z H_{5}^{0}$ leptonic channel for $M_{H_{F}} \leq 60$ (80) GeV with a data sample of $67 \mathrm{pb}^{-1}$ $\left(140 \mathrm{pb}^{-1}\right)$. Prospects for detection are therefore approximately the same as for $h$.

It is probable that the Tevatron will be upgraded in luminosity with $2 \mathrm{fb}^{-1}$ being possible by the year 2000. The increased number of events would allow heavier $M_{H_{F}}$ to be probed. For $H_{1}^{0^{\prime}}$ with $s_{H}^{2}=0.39$ one would expect $\geq 3$ events in the leptonic channel if $M_{H_{F}} \leq 110 \mathrm{GeV}$. To probe beyond this mass region requires another large increase in luminosity due to the rapid weakening of BR $\left(H_{F} \rightarrow \gamma \gamma\right)$. In Ref. [17] we suggested that the theoretical motivation for the HTM would require $s_{H} \geq 0.1\left(s_{H}^{2} \geq 0.01\right)$. For this 'minimum' value the upgraded Tevatron would produce $\geq 3$ events in the leptonic channel if $M_{H_{F}} \leq 80 \mathrm{GeV}$. Therefore the coverage would be superior to that of LEP2, the latter only being able to probe the region $M_{H_{F}} \leq \sqrt{s}-100 \mathrm{GeV}$ if $H_{F}$ has $\phi^{0}$ strength couplings. For previous searches at LEP see Refs. [27, [28].

\section{Conclusions}

We have studied the detection prospects of fermiophobic Higgs bosons $\left(H_{F}\right)$ at the Fermilab Tevatron. Such particles do not possess a tree-level coupling to fermions and can arise in various non-minimal Higgs models. Importantly, fermiophobia is not possible in the minimal supersymmetric model (MSSM) and thus searching for $H_{F}$ is well motivated. We considered the 2HDM (Model I) and the HTM in which can arise the fermiophobic bosons $H_{1}^{0^{\prime}}, h$ and $H_{5}^{0}$. The dominant decay channel for $M_{H_{F}} \leq 80$ $\mathrm{GeV}$ is $H_{F} \rightarrow \gamma \gamma$, and backgrounds are small. Such a decay has a branching ratio of the order $0.1 \%$ for the minimal SM Higgs $\left(\phi^{0}\right)$ and the lightest CP-even Higgs of the $\operatorname{MSSM}\left(h^{S U S Y}\right)$. If the $H_{F} V V(V=W$ or $Z)$ coupling is close to its maximum value then with $140 \mathrm{pb}^{-1}$ of data at the Tevatron a strong signal would be present for $H_{1}^{0^{\prime}}$ $\left(h, H_{5}^{0}\right)$ if $M_{H_{F}} \leq 90(80) \mathrm{GeV}$. It is possible to distinguish $H_{1}^{0^{\prime}}$ from $h$ and $H_{5}^{0}$ due to 
the possibility of a significantly larger cross section, although we suggested that the mere detection of a $H_{F}$ would indicate the HTM.

Prospects are improved at an upgraded Tevatron $\left(2 \mathrm{fb}^{-1}\right)$. For $H_{1}^{0^{\prime}}$ with maximum $H_{F} V V$ coupling detection is possible if $M_{H_{F}} \leq 110 \mathrm{GeV}$. This collider covers more parameter $\left(M_{H_{F}}, s_{H}\right)$ space than is possible at LEP2. For larger $M_{F}$ the decay channel $H_{F} \rightarrow \gamma \gamma$ weakens rapidly and thus the distinctive signature of $H_{F}$ becomes increasingly difficult to extract. For this higher mass region, it might be possible to distinguish $H_{F}$ from $\phi^{0}$ and $h^{S U S Y}$ due to the absence of $b \bar{b}$ decays; this branching ratio is significant for $\phi^{0}$ and $h^{S U S Y}$ if $M_{\phi^{0}} \leq 150 \mathrm{GeV}$ but negligible for $H_{F}\left(H_{F} \rightarrow W W^{*}\right.$ dominates). For still heavier $H_{F}$ (which would be in the range of the Large Hadron Collider), distinguishing would require the observation of decays of $H_{F}$ to lighter Higgs bosons e.g. $H_{F} \rightarrow A^{0} Z$, which would not be present for $\phi^{0}$. However, the latter decays are also possible for $h^{S U S Y}$. If the Tevatron is not upgraded then the Large Hadron Collider should cover the range inaccessible at LEP2 (i.e. $M_{H_{F}} \geq 80 \mathrm{GeV}$ ). Studies of detection prospects in the $\gamma \gamma$ channel for $\phi^{0}$ at this collider have been performed [29]. The conclusion is that detection is possible if a very high di-photon mass resolution can be achieved; this is partly due to $\mathrm{BR}\left(\phi^{0} \rightarrow \gamma \gamma\right) \approx 0.1 \%$. For $H_{F}$ the significantly larger $\mathrm{BR}\left(H_{F} \rightarrow \gamma \gamma\right)$ would make detection much easier as long as the production cross section is not too suppressed relative to $\phi^{0}$.

\section{Acknowledgements}

I wish to thank W.J. Stirling and S. Willenbrock for useful comments. This work has been supported by the UK EPSRC.

\section{References}

[1] S. Weinberg, Phys. Rev. Lett. 19 (1967) 1264.

[2] P.W. Higgs, Phys. Lett. B12 (1964) 132, Phys. Rev. Lett. 13 (1964) 508.

[3] J.F. Gunion, H.E. Haber, G.L. Kane and S. Dawson, The Higgs Hunter's Guide (Addison-Wesley, Reading, 1990).

[4] H. Haber, G. Kane and T. Sterling, Nucl. Phys. B161 (1979) 493.

[5] H. Pois, T. Weiler and T.C. Yuan, Phys. Rev. D47 (1993) 3886.

[6] D.A. Ross and M. Veltmann, Nucl. Phys. B95 (1975) 135.

[7] H. Georgi and M. Machacek, Nucl. Phys. B262 (1985) 463. 
[8] J.F. Gunion, R. Vega, and J. Wudka, Phys. Rev. D42 (1990) 1673; Phys. Rev. D43 (1991) 2322.

[9] P. Bamert and Z. Kunszt, Phys. Lett. B306 (1993) 335.

[10] V. Barger, J.L. Hewett and R.J.N. Phillips, Phys. Rev. D41 (1990) 3421.

[11] S.L. Glashow and S. Weinberg, Phys. Rev. D15 (1977) 1958.

[12] P. Fayet, Nucl. Phys. B90 (1975) 104.

[13] A.G. Akeroyd and W.J. Stirling, Nucl. Phys. B447 (1995) 3.

[14] A.G. Akeroyd, preprint DTP 95/70 (1995) (hep-ph 9509203).

[15] K. Cheung, R.J.N. Phillips and A. Pilaftsis, Phys. Rev. D51 (1995) 4731.

[16] R. Godbole, B.Mukhopadhyaya and M. Nowakowski, Phys. Lett. B352 (1995) 388.

[17] A.G. Akeroyd, Phys. Lett. B353 (1995) 519.

[18] Y. Grossman, Nucl. Phys. B426 (1994) 355.

[19] J. Ellis, M.K. Gaillard and D.V. Nanopoulos, Nucl. Phys. B106 (1976) 292.

[20] A. Stange, W. Marciano and S. Willenbrock, Phys. Rev. D49 (1994) 1354.

[21] M.D. Diaz and T.J. Weiler, preprint VAND-TH-94-1 (1994).

[22] H. Georgi, S. Glashow, M. Machacek and D.V. Nanopoulos, Phys. Rev. Lett 40 (1978) 692.

[23] J. Ng and P. Zakarauskas, Phys. Rev. D29 (1984) 876.

[24] Z. Kunszt, Nucl. Phys. B247 (1984) 339.

[25] S.L. Glashow, D.V. Nanopoulos and A. Yildiz, Phys. Rev. D18 (1978) 1724.

[26] R. Cahn and S. Dawson, Phys. Lett. B136 (1984) 196.

[27] D. Buskulic et al., Phys. Lett. B308, 425 (1993); OPAL Collaboration, P. Acton et al., Phys. Lett. B311, 391 (1993).

[28] V. Barger, N.G. Deshpande, J.L. Hewett, and T.G. Rizzo, preprint MAD-PH-728 (1992) (hep-ph 9211234).

[29] Proceedings of the 'Large Hadron Collider Workshop', Aachen, 4-9 October 1990, eds. G. Jarlskog and D. Rein, Report CERN 90-10, ECFA 90-133, Geneva, 1990. 